\title{
Analysis of Global Sagittal Postural Patterns in Asymptomatic Chinese Adults
}

\author{
Panpan $\mathrm{Hu}^{*}$, Miao Yu*, Zhuoran Sun, Weishi Li, Liang Jiang, \\ Feng Wei, Xiaoguang Liu, Zhongqiang Chen, Zhongjun Liu \\ Department of Orthopedics, Peking University Third Hospital, Beijing, China
}

\begin{abstract}
Study Design: A prospective imaging study.
Purpose: To characterize the distribution of the global sagittal postural patterns in asymptomatic Chinese adults using Roussouly classification.

Overview of Literature: The norms of sagittal parameters in asymptomatic Chinese population have been previously described, but no report described their global sagittal postural patterns as characterized by Roussouly classification.

Methods: A cohort of 272 asymptomatic Chinese adults was recruited. Data was assimilated by reviewing the films for each subject. Sagittal parameters were measured and sagittal postural patterns were then determined according to Roussouly classification. The pattern distributions were compared across genders within the study cohort. We also compared the data across different ethnicities from our study and a previous study to further characterize Chinese sagittal postures.

Results: The cohort included 161 males and 111 females, with mean age of $23.2 \pm 4.4$ years. The average descriptive results were as below: pelvic incidence (PI) $46.4^{\circ} \pm 9.6^{\circ}$, thoracic kyphosis (TK) $24.2^{\circ} \pm 9.0^{\circ}$, lumbar lordosis (LL) $50.6^{\circ} \pm 10.6^{\circ}$, sacral slope (SS) $37.2^{\circ} \pm 7.6^{\circ}$, pelvic tilt (PT) $9.4^{\circ} \pm 6.8^{\circ}$, spinosacral angle (SSA) $131.1^{\circ} \pm 7.5^{\circ}$ and sagittal vertical axis (SVA) $17.24 \pm 32.36 \mathrm{~mm}$. Despite a significant difference between two genders in LL, PI, SSA, and SVA, no difference was found in the distribution of Roussouly types among them. $47.8 \%$ of our cohort belonged to Roussouly type 3, while type 1,2 and 4 comprised $23.2 \%, 14.0 \%$ and $15.1 \%$ of the subjects, respectively. Roussouly classification was capable of categorizing sagittal parameters except for the PT. This study also found that $4.4 \%$ of the recruited subjects belonged to the C7-anterior subgroup.

Conclusions: From a characterization of the sagittal postural patterns of asymptomatic Chinese adults using Roussouly classification, the distribution was similar between Chinese males and females; however, from a cross-study comparison, it was different between asymptomatic Chinese and Caucasian adults, with a higher proportion of Roussouly type 3 in Chinese adults.
\end{abstract}

Keywords: Sagittal postural patterns; Asymptomatic Chinese adults; Roussouly classification; Comparison; Caucasian adults

\section{Introduction}

Recently, much work has been done to elucidate the global sagittal postural patterns of different populations [1-6].
Roussouly et al. [1] employed a four-type classification in a study of 160 asymptomatic European adults, of which type 3 was perceived as a well-balanced neutral position, and the other three types were non-neutral. The classifica-

Received Jun 9, 2015; Revised Jul, 4, 2015; Accepted Jul 6, 2015

Corresponding author: Xiaoguang Liu

Department of Orthopedics, Peking University Third Hospital, No. 49 Huayuanbei Rd, Haidian District, Beijing 100191, China

Tel: +86-188-1177-8018, Fax: +86-10-8226-7368, E-mail: puth_lxg@163.com

*The first two authors contributed equally to this work. 
tion was mainly based on sacral and lumbar alignments such as sacral slope (SS), lumbar lordosis (LL), numbers of lumbar vertebrae in lordosis, inflection point and lumbar tilt (Fig. 1). Roussouly et al. [1] found that only $37.5 \%$ of asymptomatic Caucasian adults were of type 3 . Afterwards, Mac-Thiong et al. [2] characterized the global sagittal balance in a larger cohort of asymptomatic Caucasian adults, but using a new six-type classification system, which was based on the relative position of $\mathrm{C} 7$ plumbline with respect to the midpoint of upper sacral endplate and hip axis (HA, the midpoint of the line connecting bicoxofemoral centers), and found that $14.2 \%$ of asymptomatic Caucasian adults stood with C7 plumbline ahead of HA and midpoint of superior endplate of $\mathrm{S} 1$.

By categorizing the global sagittal patterns according to

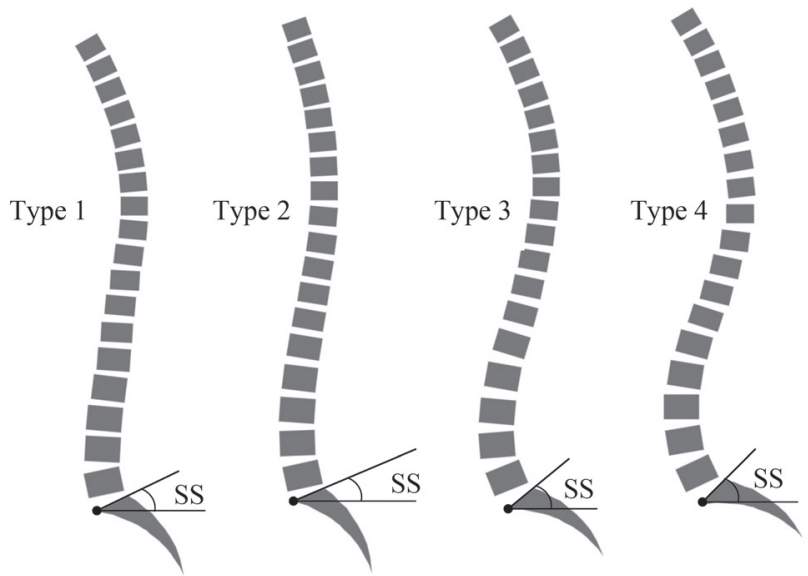

Fig. 1. Representative drawings of Roussouly types. Type 1 Sacral slope (SS) $<35^{\circ}$, apex of lumbar lordosis (LL) at middle $L 5$, the spine is hypolordotic and relatively normokyphotic; type $2 \mathrm{SS}<35^{\circ}$, apex of $\mathrm{LL}$ at base $\mathrm{L} 4$, the spine is hypolordotic and hypokyphotic; type 3 $35^{\circ}<S S<45^{\circ}$, apex of $L L$ at middle $L 4$, the spine is well balanced; type $4 \mathrm{SS}>45^{\circ}$, apex of $L L$ at base $L 3$, the spine is hyperlordotic and hyperkyphotic.
Roussouly classification, Hu et al. [6] found that $42.4 \%$ of the scoliotic Chinese adolescents belonged to type 3, in a study among 184 patients with adolescent idiopathic scoliosis. However, there has been a lack of studies exploring the global sagittal postural patterns in asymptomatic Chinese adults, though Zhu et al. [7] once characterized the norms of each sagittal parameter without the involvement of the global sagittal patterns. Thus, we designed this prospective imaging study, aiming to characterize the global sagittal postures of asymptomatic Chinese adults, and meanwhile, compared it with the Caucasian counterparts $[1,2]$.

\section{Materials and Methods}

\section{Materials}

A cohort of 272 asymptomatic Chinese adults (including 161 males and 111 females, with an average age of $23.2 \pm 4.4$ years, ranging from 18 to 45 years old) was prospectively recruited (Table 1 ). The subjects were mainly comprised of students, doctors, nurses and other employees in our hospital. The study protocol was approved by the Institutional Review Board and consent was obtained from each subject. In addition the medical history of each subject was made available. The subjects who had any of the following conditions were excluded: (1) any current and previous symptoms suggestive of spinal or orthopedic diseases, such as chronic low back pain; (2) any abnormal findings during film reviewing, such as scoliosis, spondylolisthesis, spondylolysis, Scheuermann disease, severe wedged vertebrae beyond the normal range of variation and leg discrepancy; and (3) history of medical procedure done on the spine, pelvis or lower extremities.

Table 1. Values of sagittal parameters for the entire cohort

\begin{tabular}{lccc} 
Variable & Mean & Standard deviation & $95 \%$ Confidence interval \\
\hline Age $(y r)$ & 23.2 & 4.4 & $(22.7,23.7)$ \\
\hline Thoracic kyphosis $\left(^{\circ}\right)$ & 24.2 & 9.0 & $(23.1,25.3)$ \\
\hline Lumbar lordosis $\left(^{\circ}\right)$ & 50.6 & 10.6 & $(49.3,51.9)$ \\
Sacral slope $\left(^{\circ}\right)$ & 37.2 & 7.6 & $(36.3,38.1)$ \\
\hline Pelvic incidence $\left(^{\circ}\right)$ & 46.4 & 9.6 & $(45.3,47.6)$ \\
\hline Pelvic tilt $\left({ }^{\circ}\right)$ & 9.4 & 6.8 & $(8.6,10.3)$ \\
Spinosacral angle $\left(^{\circ}\right)$ & 131.1 & 7.5 & $(130.2,132.0)$ \\
Sagittal vertical axis $(\mathrm{mm})$ & 17.24 & 32.36 & $(13.38,21.10)$ \\
\hline
\end{tabular}




\section{Methods}

\section{1) Imaging protocols}

Full-length posteroanterior (PA) and lateral X-rays of the spine were taken at our hospital. For $t$ subsequent comparison with Caucasian adults reported by Roussouly et al. [1], this study adopted the same radiographic protocol as in their study. Specifically, the subjects stood in an erect comfortable position with their hands flexed at $45^{\circ}$ to $60^{\circ}$, resting on an adjustable supports (Fig. 2), and gazed horizontally in order to reduce any inaccuracy caused by head motion, and exposures were taken from the base of the skull to the proximal femora in the left to right lateral

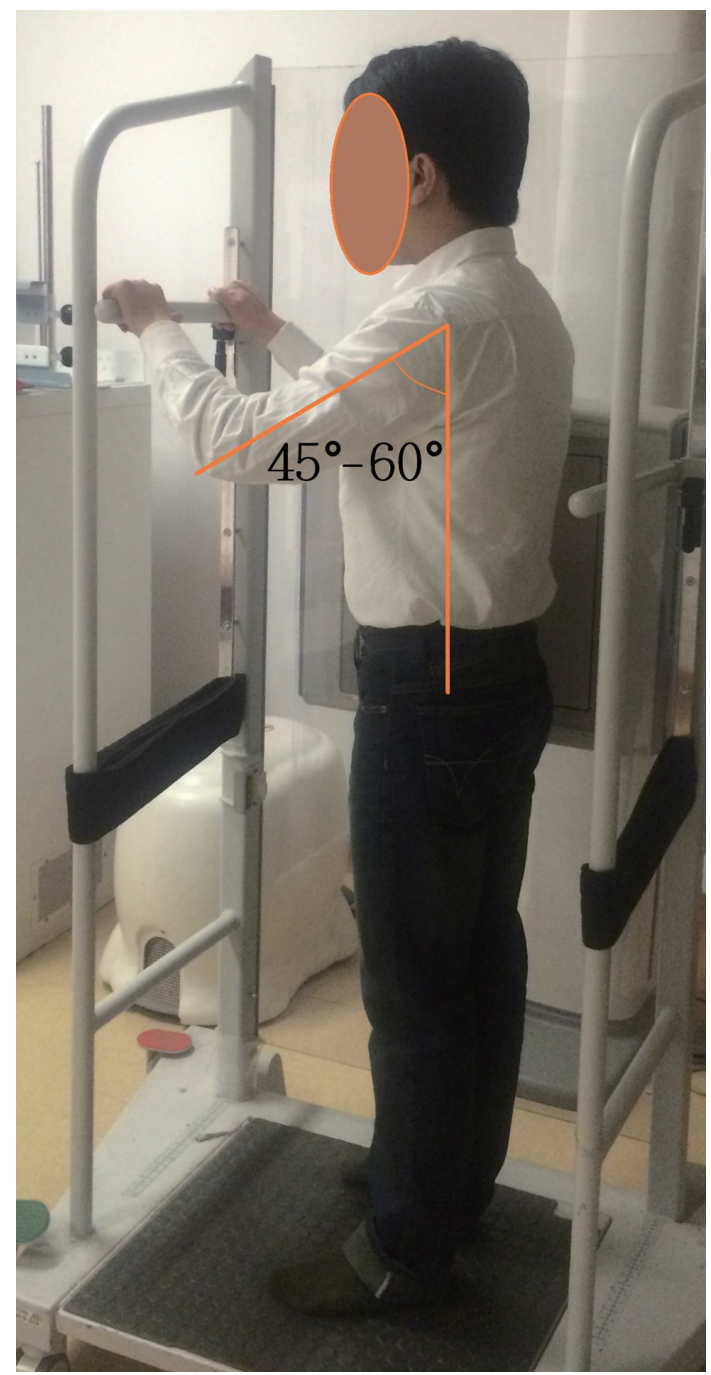

Fig. 2. The stance when taking lateral films. Subjects stood in an erect comfortable posture and were required to have their arms flexed at a consistent angle, which was about 45 to 60 degrees, by adjusting the height of a bar to support hands. plane. The distance from the radiographic source to the film was maintained at $180 \mathrm{~cm}$ and the edges of the films were squared with respect to the horizontal and vertical axes. The films were digitized by a commercially available optical scanner (GE Discovery XR650, GE Healthcare, Waukesha WI, USA). All morphologic data were archived via Picture Archiving and Communication Systems (PACS, GE Healthcare). Then the data were retrieved and measured through PACS in diagonally 20 -inch screens, with the resolution at $75 \mathrm{dpi}$.

\section{2) Parameters to measure}

The sagittal spinopelvic parameters to measure included pelvic incidence (PI), the angle subtended by the line connecting HA to the midpoint of upper sacral endplate and the line perpendicular to upper sacral endplate; SS, the angle subtended by the horizontal line and upper sacral endplate; pelvic tilt (PT), the angle subtended by the vertical line and the line connecting HA to the midpoint of upper sacral endplate; spinosacral angle (SSA), the angle subtended by the upper sacral endplate and the line from the center of $\mathrm{C} 7$ vertebral body to the midpoint of upper sacral endplate; LL, the angle subtended by the superior endplates of L1 and S1; thoracic kyphosis (TK), the angle subtended by the superior endplate of T5 and inferior endplate of T12; and sagittal vertical axis (SVA), the horizontal distance between $\mathrm{C} 7$ plumbline and the posterosuperior corner of S1, positive if C7 plumbline is behind the posterosuperior corner of S1, or otherwise negative (Fig. 3). On PA films, any significant curve (namely Cobb angles of more than $10^{\circ}$ ) was marked out for exclusion. All values were measured two times and the average results were obtained.

All subjects were categorized by Roussouly classification according to their pelvic and lumbar alignments (Fig. 1). The data on Caucasian adults for ethnical comparison were retrieved from Roussouly's study [1], in which the subjects were categorized with the same standards as in this study. Also the position of C7 plumbline was decided with respect to $\mathrm{HA}$ and the midpoint of upper sacral endplate and then Mac-Thiong's classification [2] was also applied.

\section{3) Statistical analysis}

Statistical analyses were conducted with IBM SPSS ver. 20.0 (SPSS Inc., Chicago IL, USA). Descriptive statistics were provided in the form of mean \pm standard deviation 
and the confidence interval was set at 95\% (95\% CI). Lilliefors test, which is an adaptation of KolmogorovSmirnov test, was used to examine whether the param-
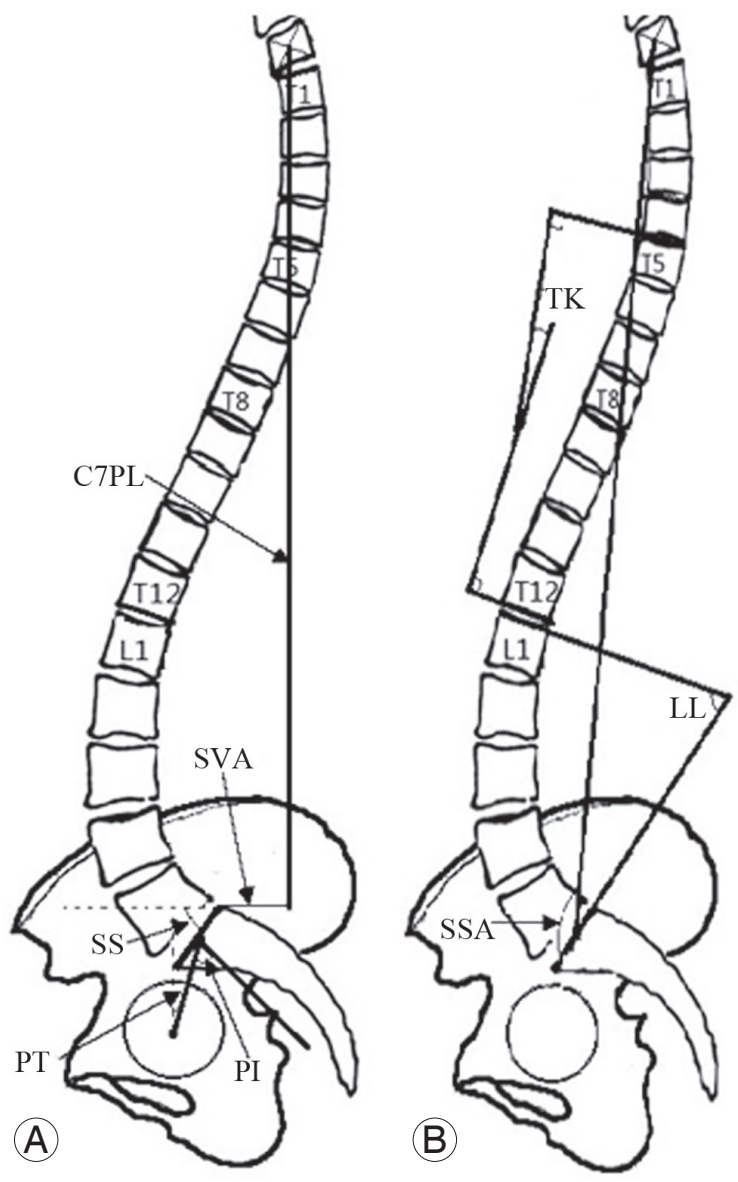

Fig. 3. Representative drawings of spinopelvic parameters. (A) PI stands for pelvic incidence; PT, pelvic tilt; SS, sacral slope; SVA, sagittal vertical axis; C7PL, C7 plumbline. (B) TK stands for thoracic kyphosis; LL, lumbar lordosis; SSA, spinosacral angle. eters were normally distributed. One-way analysis of variance test (Scheffe's method for the post hoc test), twotailed unpaired student $t$ test, and two-tailed Pearson's $\chi^{2}$ test (or two-tailed $G$-test) were utilized to compare between different groups. Statistical significance was set at 0.05 .

\section{Results}

No difference was detected in average age between the two genders $(p=0.917)$. There were significant differences in the values of LL, PI, SSA and SVA between males and females $(p<0.05)$, but no differences in TK, SS and PT $(p>0.05)$ (Table 2).

From the Chinese subjects, $47.8 \%$ belonged to Roussouly type 3 (Table 3 ). There were also 63, 38 and 41 subjects in type 1, 2 and 4 groups, respectively. Roussouly type 3 subjects were $44.7 \%$ of males and $52.3 \%$ of females (Table 3 ). No difference was found between males and females in the distribution of Roussouly types $(p=0.130)$ (Table 3 ), or in the proportions of the neutral postures $(p=0.222)$ (Table 4$)$.

All sagittal parameters except PT were significantly different among four Roussouly types $(p<0.05)$. LL, SS, PI and SSA parameters were different for all the Roussouly groups with the exception of Roussouly type 1 and type 2. There were no significant differences in PT between any two different Roussouly types. There were also none in SVA except between type 1 and type 4 groups $(p<0.05)$ (Table 5).

Besides the Roussouly classification, Mac-Thiong's classification was also applied and $4.4 \%$ of the Chinese subjects belonged to the C7-anterior subgroup (Table 6).

Table 2. Comparison of sagittal parameters between males and females

\begin{tabular}{|c|c|c|c|}
\hline Variable & Males & Females & $p$-value \\
\hline Age (yr) & $23.2 \pm 3.4$ & $23.2 \pm 5.4$ & 0.917 \\
\hline Thoracic kyphosis $\left({ }^{\circ}\right)$ & $24.6 \pm 9.4$ & $23.5 \pm 8.5$ & 0.315 \\
\hline Lumbar lordosis $\left({ }^{\circ}\right)$ & $49.4 \pm 10.7$ & $52.3 \pm 10.3$ & $0.025^{\mathrm{a})}$ \\
\hline Sacral slope $\left({ }^{\circ}\right)$ & $36.9 \pm 7.9$ & $37.7 \pm 7.2$ & 0.368 \\
\hline Pelvic incidence $\left({ }^{\circ}\right)$ & $45.3 \pm 9.7$ & $48.1 \pm 9.2$ & $0.021^{\mathrm{a})}$ \\
\hline Pelvic tilt $\left({ }^{\circ}\right)$ & $8.8 \pm 7.1$ & $10.4 \pm 6.3$ & 0.066 \\
\hline Spinosacral angle $\left({ }^{\circ}\right)$ & $130.0 \pm 7.4$ & $132.8 \pm 7.4$ & $0.002^{\mathrm{al}}$ \\
\hline Sagittal vertical axis (mm) & $11.83 \pm 33.24$ & $25.09 \pm 29.44$ & $0.001^{a)}$ \\
\hline
\end{tabular}

Values are presented as mean \pm standard deviation.

${ }^{\text {a) }}$ Statistically significant at $p<0.05$, unpaired student $t$ test (two-tailed). 
Table 3. Distribution of Roussouly classification for the entire cohort and different genders

\begin{tabular}{lccc} 
Roussouly types & All $(\mathrm{n}=272)$ & Male $(\mathrm{n}=161)$ & Female $(\mathrm{n}=111)$ \\
Type 1 & $63(23.2)$ & $40(24.8)$ & $23(20.7)$ \\
Type 2 & $38(14.0)$ & $28(17.4)$ & $10(9.0)$ \\
\hline Type 3 & $130(47.8)$ & $72(44.7)$ & $58(52.3)$ \\
Type 4 & $41(15.1)$ & $21(13.0)$ & $20(18.0)$ \\
\hline -value & Not applicable & $0.130^{\text {a) }}$ & \\
\hline
\end{tabular}

Values are presented as number (\%).

${ }^{\text {a) }}$ Comparison between the two genders generated no significant difference $(p>0.05)$, using two-tailed Pearson's $\chi^{2}$ test.

Table 4. Sagittal alignments in different genders

\begin{tabular}{lccc} 
& \multicolumn{3}{c}{ Sagittal alignments } \\
\cline { 2 - 3 } & Neutral & Non-neutral & Total \\
Male & $72(44.7)$ & $89(55.3)$ & 161 \\
Female & $58(52.3)$ & $53(47.7)$ & 111 \\
\hline
\end{tabular}

Values are presented as number (\%).

$p=0.222, \chi^{2}=1.494, \mathrm{df}=1$, Pearson's $\chi^{2}$ test (two-tailed).

Table 5. Sagittal parameters in different Roussouly types

\begin{tabular}{|c|c|c|c|c|c|c|c|c|c|c|}
\hline \multirow[t]{2}{*}{ Parameter } & \multirow[t]{2}{*}{ Type 1} & \multirow[t]{2}{*}{ Type 2} & \multirow[t]{2}{*}{ Type 3} & \multirow{2}{*}{ Type 4} & \multicolumn{6}{|c|}{$\begin{array}{l}p \text {-values for the comparison } \\
\text { of any two Roussouly types }\end{array}$} \\
\hline & & & & & 1 vs. 2 & 1 vs. 3 & 1 vs. 4 & 2 vs. 3 & 2 vs. 4 & 3 vs. 4 \\
\hline Thoracic kyphosis ( $\left(^{\circ}\right)$ & $24.1 \pm 8.9$ & $16.4 \pm 8.7$ & $26.2 \pm 8.4$ & $25.3 \pm 7.5$ & $0.000^{a)}$ & 0.488 & 0.921 & $0.000^{a)}$ & $0.000^{a)}$ & 0.959 \\
\hline Lumbar lordosis $\left({ }^{\circ}\right)$ & $46.8 \pm 6.3$ & $38.8 \pm 5.9$ & $53.5 \pm 6.7$ & $65.1 \pm 5.8$ & $0.005^{a)}$ & $0.000^{\mathrm{a})}$ & $0.000^{\mathrm{a})}$ & $0.000^{a)}$ & $0.000^{a)}$ & $0.000^{a)}$ \\
\hline Sacral slope $\left({ }^{\circ}\right)$ & $29.8 \pm 4.2$ & $31.4 \pm 2.8$ & $38.9 \pm 2.8$ & $51.3 \pm 4.3$ & 0.601 & $0.000^{\mathrm{al}}$ & $0.000^{\mathrm{al}}$ & $0.000^{a)}$ & $0.000^{a)}$ & $0.000^{a)}$ \\
\hline Pelvic incidence $\left({ }^{\circ}\right)$ & $40.3 \pm 7.3$ & $43.1 \pm 8.6$ & $47.6 \pm 6.4$ & $59.9 \pm 9.8$ & 0.738 & $0.000^{\mathrm{a})}$ & $0.000^{\mathrm{a})}$ & 0.308 & $0.000^{a)}$ & $0.000^{a)}$ \\
\hline Pelvic tilt $\left({ }^{\circ}\right)$ & $10.5 \pm 6.9$ & $11.7 \pm 6.7$ & $8.6 \pm 6.5$ & $8.5 \pm 9.6$ & 0.908 & 0.259 & 0.468 & 0.951 & 0.964 & 1.000 \\
\hline $\begin{array}{l}\text { Spinosacral } \\
\text { angle }\left({ }^{\circ}\right)\end{array}$ & $125.3 \pm 4.8$ & $124.7 \pm 3.5$ & $132.6 \pm 4.8$ & $142.5 \pm 4.6$ & 0.985 & $0.000^{\mathrm{a})}$ & $0.000^{a)}$ & $0.000^{a)}$ & $0.000^{a)}$ & $0.000^{a)}$ \\
\hline $\begin{array}{l}\text { Sagittal vertical } \\
\text { axis }(\mathrm{mm})\end{array}$ & $29.71 \pm 30.16$ & $11.17 \pm 34.05$ & $15.44 \pm 36.14$ & $-3.05 \pm 29.73$ & 0.456 & 0.281 & $0.007^{\mathrm{al}}$ & 0.983 & 0.705 & 0.162 \\
\hline
\end{tabular}

Values are presented as mean \pm standard deviation.

a) Statistically significant at $p<0.05$, one-way analysis of variance test (Scheffe method for the post hoc test).

Type 6. Distribution of the global sagittal balance for the entire cohort ${ }^{\text {a) }}$

\begin{tabular}{lc} 
Type & $n(\%)$ \\
\hline Type 1 & $214(78.7)$ \\
Type 2 & $32(11.8)$ \\
Type 3 & $6(2.2)$ \\
Type 4 & $14(5.1)$ \\
\hline Type 5 & 0 \\
Type 6 & $6(2.2)$ \\
\hline
\end{tabular}

${ }^{a}$ The cohort was grouped according to Mac-Thiong's definition [2], and Type 3 and Type 6 belonged to C7-anterior subgroup.

\section{Discussion}

This prospective study focused on the categorization of the global sagittal postures of asymptomatic Chinese adults in order to provide some references for future studies. For a comparison between the Caucasian and Chinese ethnicities, we deliberately adopted a radiographic protocol as closely matched to that of Roussouly's as possible (see "Imaging protocols"). In addition, this study bifurcated the cohort according to gender in order to reveal 
the divergence in the distribution of the sagittal postural patterns between males and females. To the best of our knowledge, this study was the first to thoroughly explore the distribution of the global sagittal postural patterns in Chinese adults and compare it with that with the Caucasian counterparts.

In total, 272 asymptomatic adult were enrolled, whose average PI value was $46.4^{\circ} \pm 9.6^{\circ}$ (Table 1 ), lower than that of Caucasian adults $\left(51.7^{\circ}\right.$ to $\left.55.0^{\circ}\right)[1,2,8]$. According to various publications, this phenomenon might be related to the smaller average body size of Chinese population than that of the Caucasian adults [7,9]. Our study found that $47.8 \%$ of asymptomatic Chinese adults possessed a neutral sagittal posture, namely Roussouly type 3 , which was higher than $37.5 \%$ of Caucasian adults, as reported by Roussouly et al. [1]. As we adopted an identical imaging protocol reported by Roussouly et al. [1], this difference between the two ethnics could not be attributed to nuances of imaging protocols and measurements. It implies that Chinese adults might have a lower susceptibility to clinical entities like low back pain, according to the previous studies on the relationship between Roussouly types and low back pain $[4,10]$.

In our study of asymptomatic individuals, females had higher SVA and SSA values than males (Table 2) as females had a spine more prone to a backward drift, compared with males, and this was reflected by a higher proportion of Roussouly type 3 and type 4's in females (Table 3). According to Araujo et al. [4] study, increased SVA in Roussouly type 4 were associated with pain and decreased quality of life measures. This finding implied that females were at a higher risk to develop certain spinal pathologies. However, our study also found that Chinese females possessed a favorable distribution of neutral sagittal postural pattern (Table 4), a pattern that is inversely related to low back pain as demonstrated by Chaleat-Valayer et al.s [10] study. To resolve these conflicting claims, future studies may have to revisit the topic.

Nearly all the sagittal parameters were significantly different between Roussouly types except PT (Table 6), and this is a reflection of the effectiveness of Roussouly classification on sagittal alignments. However we observed that in addition to PT, SVA was similar between any two Roussouly types except between type 1 and type 4 groups (Table 6). According to a previous publication [4], these two parameters, PT and SVA, have a stronger association with quality of life measures than other parameters. As
Roussouly's classification cannot stratify PT and SVA, it implies a limited applicability of this metric for quality of life measures.

Within the Mac-Thiong's six-type classification [2], the first three types were defined as the midpoint of upper sacral endplate behind HA, whereas type 4, 5, and 6 were defined as the midpoint ahead of HA. Type 1 and 4 referred to subjects with $\mathrm{C} 7$ plumbline behind both the midpoint and HA, type 2 and 5 between the midpoint and HA, and type 3 and 6 ahead of both the midpoint and HA. Unfortunately, the classification does not address which types are the neutral or balanced global sagittal alignments. The subjects of our study were divided into six types accordingly, and the result was different from that of Caucasian adults [2]. There were $78.7 \%$ of Chinese adults in type 1 versus $55 \%$ for Caucasian adults. Since there have been no risk or correlation analyses regarding the relationship of this classification with clinical pathologies and/or future prognoses, this distributive difference between the two ethnicities could not provide for a direct clinical conclusion on any posture benefits for sagittal balance. Nevertheless, the forward displacement of C7 plumbline relative to sacrum may be thought of an increased risk in developing a spinal pathology $[2,4]$. Therefore with $4.4 \%$ of Chinese adults standing with the forward displacement of C7 plumbline compared with $14.2 \%$ of Caucasian adults [2], it might indicate a lower risk of spinal pathology for asymptomatic Chinese adults. To verify this hypothesis, it is necessary to design an appropriate correlation study with risk analysis and longitudinal followups.

There were some limitations in our prospective study. First, the recruitment bias seemed inevitable, considering the geographical origin of the subjects and the younger mean age of the enrolled subjects in this study, which could possibly compromise the validity of the comparison between the two ethnicities. Our cohort, however, had a comparable subject source to that of Roussouly's [1] study, and it partially justifies a cross comparison analysis of the two ethnicities. Finally, as this was not a confirmatory study, any clinical interpretations need to be supported by future studies.

\section{Conclusions}

Enrolling 272 asymptomatic Chinese adults, $23.2 \pm 4.4$ years old ranging from 18 to 45 years, this prospective 
study found that $47.8 \%$ of asymptomatic Chinese adults possessed a sagittal posture of Roussouly type 3, higher than that of Caucasian adults, and that Chinese males and females had a similar distribution of the sagittal postural patterns. Also there were fewer Chinese adults standing with C7 plumbline in front of sacrum. Overall, a comparison of the global sagittal posture patterns between the two ethnicities seems to indicate a favorable result for the Chinese men and women in this age range.

\section{Conflict of Interest}

No potential conflict of interest relevant to this article was reported.

\section{References}

1. Roussouly P, Gollogly S, Berthonnaud E, Dimnet J. Classification of the normal variation in the sagittal alignment of the human lumbar spine and pelvis in the standing position. Spine (Phila Pa 1976) 2005;30:346-53.

2. Mac-Thiong JM, Roussouly P, Berthonnaud E, Guigui P. Sagittal parameters of global spinal balance: normative values from a prospective cohort of seven hundred nine Caucasian asymptomatic adults. Spine (Phila Pa 1976) 2010;35:E1193-8.

3. Lee JH, Kim KT, Suk KS, et al. Analysis of spinopelvic parameters in lumbar degenerative kyphosis: cor- relation with spinal stenosis and spondylolisthesis. Spine (Phila Pa 1976) 2010;35:E1386-91.

4. Araujo F, Lucas R, Alegrete N, Azevedo A, Barros $H$. Sagittal standing posture, back pain, and quality of life among adults from the general population: a sex-specific association. Spine (Phila Pa 1976) 2014;39:E782-94.

5. Yu M, Silvestre C, Mouton T, Rachkidi R, Zeng L, Roussouly P. Analysis of the cervical spine sagittal alignment in young idiopathic scoliosis: a morphological classification of 120 cases. Eur Spine J 2013;22:2372-81.

6. Hu P, Yu M, Liu X, Zhu B, Liu X, Liu Z. Analysis of the relationship between coronal and sagittal deformities in adolescent idiopathic scoliosis. Eur Spine J 2016;25:409-16.

7. Zhu Z, Xu L, Zhu F, et al. Sagittal alignment of spine and pelvis in asymptomatic adults: norms in Chinese populations. Spine (Phila Pa 1976) 2014;39:E1-6.

8. Vaz G, Roussouly P, Berthonnaud E, Dimnet J. Sagittal morphology and equilibrium of pelvis and spine. Eur Spine J 2002;11:80-7.

9. Li WS, Sun ZR, Chen ZQ. Radiographic analysis of sagittal spino-pelvic alignment in asymptomatic Chinese adults. Chin J Orthop 2013;33:447-53.

10. Chaleat-Valayer E, Mac-Thiong JM, Paquet J, Berthonnaud E, Siani F, Roussouly P. Sagittal spinopelvic alignment in chronic low back pain. Eur Spine J 2011;20 Suppl 5:634-40. 